\title{
Limits and accuracy of valence force field models for $\operatorname{In}_{x} \mathrm{Ga}_{1-x} \mathrm{~N}$ alloys
}

\author{
Frank Grosse* and Jörg Neugebauer \\ Fritz-Haber-Institut der Max-Planck-Gesellschaft, Faradayweg 4-6, D-14195 Berlin (Dahlem), Germany
}

(Received 6 June 2000; published 7 February 2001)

\begin{abstract}
Formation energies, equilibrium geometries, and elastic properties of ordered $\operatorname{In}_{x} \mathrm{Ga}_{1-x} \mathrm{~N}$ structures have been calculated employing density funcional theory. Based on these results, limits and accuracy of several valence force field (VFF) models are compared. While these empirical models have been shown to work reasonably well to describe zincblende III/V semiconductors we find significant deviations for group III nitrides $(\mathrm{GaN}, \mathrm{InN})$ and their alloys. We therefore propose a new model that correctly takes into account the long-range electrostatic interactions. Although only the elastic constants of the binary zincblende compounds and the formation energy difference to wurtzite are used as input, the model correctly describes the formation energies and structure of wurtzite binary compounds and ternary alloys.
\end{abstract}

DOI: 10.1103/PhysRevB.63.085207

PACS number(s): 61.50.Ah, 61.50.Lt, 81.05.Ea, 61.82.Fk

\section{INTRODUCTION}

The change of concentration $x$ in a ternary alloy system $\mathrm{A}_{x} \mathrm{~B}_{1-x} \mathrm{C}$ enables in principle continuous tuning of any physical property given by that of the binary constituents AC and BC. A technologically important example is band gap engineering where the width of the band gap is controlled by the alloy composition $x$. A new and technologically very interesting semiconductor alloy system where this effect is utilized is $\operatorname{In}_{x} \mathrm{Ga}_{1-x} \mathrm{~N}$. This alloy is used, e.g., as an active region in $\mathrm{GaN}$-based optoelectronic devices and (assuming complete miscibility) allows to tune the band gap between $1.9 \mathrm{eV}(\mathrm{InN})$ and $3.5 \mathrm{eV}(\mathrm{GaN})$, i.e., between the infrared and ultraviolet region of the optical spectrum. An important issue for $\operatorname{In}_{x} \mathrm{Ga}_{1-x} \mathrm{~N}$ alloys is homogeneity: Experimentally it has been found that these alloys are often unstable against phase separation, spatial fluctuations in the In concentration, or partial ordering. ${ }^{1-4}$ These effects have been shown to strongly affect the luminescence efficiency. It is therefore crucial to get a deeper insight into the energetics and local atomic structure of $\operatorname{In}_{x} \mathrm{Ga}_{1-x} \mathrm{~N}$ alloys.

Whereas the properties of the binary compounds AlN, $\mathrm{GaN}$, and InN have been calculated with different highly accurate first-principles methods ${ }^{5-10}$ the investigation of their alloy properties is mainly based on empirical valence force field (VFF) models for atomic structure relaxation. Ho and Stringfellow ${ }^{11}$ derived a temperature/alloy composition phase diagram with this method indicating a rather large miscibility gap with a critical temperature of $1250^{\circ} \mathrm{C}$. Mattila and Zunger ${ }^{12}$ calculated the bond length distribution and atomic structure for random alloys based on the VFF model. Using alloy structures optimized within the same VFF model, Bellaiche and Zunger ${ }^{13}$ investigated the influence of short-range order on optical and electronic properties of large alloy systems employing empirical pseudopotential theory.

While VFF models have been widely used to describe the properties of several alloy systems the accuracy and reliability of these methods is hard to assess. The input parameters for the empirical VFF models have been either calculated by the delta-lattice model ${ }^{11}$ or by fitting the elastic constants of the zincblende binaries InN and GaN. ${ }^{7,14}$ Previous studies for $\mathrm{GaInP}^{15}$ alloys indicated that the accuracy of this procedure is sufficient to describe and predict alloy properties such as bond lengths and phase diagrams. For group III nitrides, however, detailed tests about the applicability and accuracy of VFF models are still lacking. A unique feature of this material system is the much larger ionicity compared to "traditional" semiconductors like GaAs and, as a consequence, the wurtzite crystal structure. Therefore, a purely elastic model like the VFF model might be inadequate to describe correctly the electrostatic contributions and is not able to distinguish between the wurtzite and zincblende crystal structure. In fact, we will show in the present paper that electrostatic contributions are essential to obtain the energetics and structure of $\operatorname{In}_{x} \mathrm{Ga}_{1-x} \mathrm{~N}$ alloys correctly.

The paper is organized as follows: In Sec. II we present and discuss our first principles calculations for $\mathrm{InN}$ and $\mathrm{GaN}$ binaries and $\mathrm{In}_{x} \mathrm{Ga}_{1-x} \mathrm{~N}$ alloys. Based on these results we discuss in Sec. III the accuracy and reliability of different VFF models such as the form given by Keating ${ }^{16}$ or its extension by Martin. ${ }^{17}$ Both have been successfully applied to describe alloys of "traditional," zincblende semiconductors. For $\operatorname{In}_{x} \mathrm{Ga}_{1-x} \mathrm{~N}$ alloys we find, however, that the VFF results significantly deviate from those obtained by our first principles calculations. We therefore describe in Sec. IV a new model, describing correctly ternary alloys and the structural and energetic differences between the zincblende and wurtzite phases of $\mathrm{GaN}$ and $\mathrm{InN}$.

\section{FIRST PRINCIPLES CALCULATIONS}

We calculated elastic properties of the binary compounds $\mathrm{GaN}$ and $\mathrm{InN}$, and the equilibrium structure and energetics of the ternary ordered $\mathrm{In}_{x} \mathrm{Ga}_{1-x} \mathrm{~N}$ structures, employing density functional theory ${ }^{18}$ (DFT) in the local density approximation and $a b$ initio norm-conserving pseudopotentials. ${ }^{19}$ We performed a great number of convergence checks to test the reliability and accuracy of the plane wave basis set, the pseudopotentials and the $k$-point sampling. These checks reveal an important aspect: the Ga $3 d$ and In $4 d$ electrons play an active role for the chemical binding in $\operatorname{In}_{x} \mathrm{Ga}_{1-x} \mathrm{~N}$ alloys and cannot be simply treated as core electrons. ${ }^{20}$ Otherwise, spurious results in the alloy formation energies or bond lengths may be obtained. The wave functions are expanded 
TABLE I. Calculated structural and elastic properties for zincblende $\mathrm{GaN}$ and $\mathrm{InN}$ in comparison with experimental values for lattice constants (Ref. 31) and with other density function calculations (PW, plane wave expansion; LMTO, linear muffin tin orbitals).

\begin{tabular}{lcccc}
\hline \hline $\begin{array}{l}\text { Zincblende } \\
\mathrm{GaN}\end{array}$ & Experiment & $\begin{array}{c}\text { Present } \\
\text { PW }\end{array}$ & $\begin{array}{c}\text { LMTO } \\
\text { Refs. 7 and } 8\end{array}$ & $\begin{array}{c}\text { PW } \\
\text { Refs. 6 and 20 }\end{array}$ \\
\hline$a(\AA)$ & & 4.517 & 4.46 & 4.460 \\
$B(\mathrm{GPa})$ & $4.52-4.55$ & 197 & 201 and 199 & 187 \\
$C_{11}^{c}(\mathrm{GPa})$ & 277 & 296 and 282 & 293 \\
$C_{12}^{c}(\mathrm{GPa})$ & 156 & 154 and 159 & 159 \\
$C_{44}^{c}(\mathrm{GPa})$ & & 206 and 142 & 155 \\
& & & \\
$\mathrm{Zincblende}$ & & & LMTO & PW \\
$\mathrm{InN}$ & & Present & Refs. 7 and 8 & Refs. 6 and 20 \\
\hline$a(\AA)$ & DFT & 5.005 & 4.92 & 4.932 \\
$B(\mathrm{GPa})$ & 4.98 & 142 & 139 & 140 \\
$C_{11}^{c}(\mathrm{GPa})$ & 178 & 184 and 182 & 187 \\
$C_{12}^{c}(\mathrm{GPa})$ & 124 & 116 and 125 & 86 \\
$C_{44}^{c}(\mathrm{GPa})$ & 82 & 177 and 79 & \\
\hline \hline
\end{tabular}

in a plane wave basis set up to an energy cutoff of $80 \mathrm{Ry}$. This high energy cutoff was found to be essential to describe the external lattice parameters for the different alloys correctly. Soft Troullier-Martins pseudopotentials are used. ${ }^{21,22}$ The $k$ integration over the Brillouin zone is performed up to a $6 \times 6 \times 4$ Monkhorst-Pack mesh. ${ }^{23}$ Details of the method can be found elsewhere. ${ }^{24,25}$

We first discuss the results for the zincblende binaries $\mathrm{GaN}$ and $\mathrm{InN}$ which will be later used as input parameters for our extended VFF model. The results for the equilibrium values (lattice constants) and for the elastic constants are listed in Table I and compared with values of previous first principles calculations. ${ }^{6-8}$ Generally, a good agreement is found. A comparison with experiment is difficult since the stable modification of $\mathrm{GaN}$ is the wurtzite phase and zincblende $\mathrm{GaN}$ can be synthesized by epitaxial growth only. For the zincblende modification the measured and the calculated lattice constant agree well. We have also calculated the structural properties of $\mathrm{GaN}$ and $\mathrm{InN}$ in the wurtzite modification. The main difference between the zincblende and wurtzite phase is the lower symmetry of the wurtzite phase. The local coordination, however, is identical up to third nearest neighbors. The results are listed in Table II and will be used later to compare the accuracy of the empirical VFF models. Further included in Table II are the experimental values for the external and internal lattice parameters. Generally, only small modifications compared to the results for the zincblende structure are found. The wurtzite phase is characterized by two inequivalent bond lengths and a $c / a$ ratio slightly below the ideal value $(=\sqrt{8 / 3})$. Furthermore the wurtzite modification has a $11 \mathrm{meV}(\mathrm{GaN})$ and $19 \mathrm{meV}$ (InN) per cation lower formation energy. The two inequivalent bond lengths differ rather little and are both close to the zincblende bond length. For GaN the values are $r_{0}=1.956 \AA$ (zincblende) and $1.955 \AA$ and $1.958 \AA$ (wurtzite). For InN these differences are even smaller: $2.167 \AA$ (zincblende) and $2.167 \AA$ and $2.168 \AA$ (wurtzite).
In order to study $\operatorname{In}_{x} \mathrm{Ga}_{1-x} \mathrm{~N}$ alloys we have studied a large number of ordered structures with varying In compositions. The In concentration has been varied from $x=0(\mathrm{GaN})$ to $x=1(\mathrm{InN})$ in steps of $\Delta x=0.065$. For all these structures we start from a wurtzite $(2 \times 2 \times 2)$ unit cell where the Ga atoms are replaced by In atoms obtaining all relevant inequivalent structures. For this specific choice of unit cell we have four cation layers (perpendicular to the [0001] axis) per cell. The chemical composition of each of these layers is shown in the first column of Tables III and IV: the lower two planes are shown in the left figure, the upper two planes in the right figure. To give a specific example, the special ordered structures in the fourth row $(x=25 \%$ or $75 \%$, respectively) of Table III are shown in Fig. 1 as a ball and stick model.

TABLE II. Calculated structural properties for wurtzite GaN and InN with DFT and ZVFF model. The experimental values are taken from Ref. 31. $\Delta E$ gives the difference of the formation enthalpies of the equilibrium zincblende and the fully relaxed wurtzite modification.

\begin{tabular}{lccc}
\hline \hline Wurzite GaN & DFT & ZVFF & Expt. \\
\hline$a(\AA)$ & 3.196 & 3.199 & 3.189 \\
$c(\AA)$ & 5.206 & 5.200 & 5.185 \\
$c / a$ (ideal: 1.633$)$ & 1.629 & 1.626 & 1.626 \\
$u$ (ideal: 0.375) & 0.376 & 0.376 & \\
$\Delta E$ (meV) & 11 & 11 & \\
& & & \\
Wurzite InN & DFT & ZVFF & Expt. \\
\hline$a(\AA)$ & 3.545 & 3.553 & 3.548 \\
$c(\AA)$ & 5.761 & 5.730 & 5.760 \\
$c / a$ (ideal: 1.633$)$ & 1.625 & 1.613 & 1.623 \\
$u$ (ideal: 0.375$)$ & 0.376 & 0.376 & \\
$\Delta E$ (meV) & 19 & 19 & \\
\hline \hline
\end{tabular}


TABLE III. Comparison of structures with lowest energy for different concentrations. The structure is schematically drawn as a view along [0001], so that only the cations can be seen. For $25 \%$ $(75 \%)$ the ordered $(2 \times 2 \times 2)$ unit cell is given in Fig. 1 .

\begin{tabular}{cccc}
\hline Structure & $x(\%)$ & Unit cell & $\Delta H(\mathrm{meV})$ \\
\hline & $6.25,93.75$ & $2 \times 2 \times 2$ & 28,24 \\
& $12.5,87.5$ & $2 \times 2 \times 1$ & 46,37 \\
& $18.75,81.25$ & $2 \times 2 \times 2$ & 58 \\
& 25,75 & $2 \times 2 \times 1$ & 57,36 \\
& $37.5,62.5$ & $2 \times 2 \times 1$ & 79,70 \\
& 50.0 & $2 \times 1 \times 1$ & 63 \\
\hline
\end{tabular}

The equilibrium geometry for each ordered structure has been obtained by carefully optimizing both the external degrees of freedom (the lateral lattice constant $a$ and the $c / a$ ratio) and all internal coordinates. On the basis of these results we obtain the formation enthalpy of the different ordered structures defined as

$$
\Delta H(x)=E_{\mathrm{tot}}(x)-(1-x) E_{\mathrm{tot}}^{\mathrm{GaN}}-x E_{\mathrm{tot}}^{\mathrm{InN}} .
$$

Here $E_{\mathrm{tot}}(x)$ is the total energy for a specific ordered structure with an In concentration $x . E_{\text {tot }}^{\mathrm{GaN}}$ and $E_{\text {tot }}^{\mathrm{InN}}$ represent the total energies for bulk $\mathrm{GaN}$ and $\mathrm{InN}$, respectively. For a fixed concentration we compare different arrangements of $\mathrm{Ga}$ and In atoms within the $(2 \times 2 \times 2)$ unit cell to find the ordered structure with the lowest formation enthalpy. These results (structure, formation enthalpy) are summarized in Table III whereas Table IV contains the calculated formation enthalpies for fixed concentrations $(25 \%, 75 \%)$ but different arrangements. A graphical representation is given in Figs. 2-4 together with results of different VFF approaches which will be discussed below.

A striking result of the first principles calculations is the large dependence of the formation enthalpy with respect to the specific structure. For the same macroscopic chemical composition $x$, but different atomic arrangements, differences of more than $100 \%$ in the alloy formation energy are found. A first analysis of these results revealed that cations of the same species repel each other. ${ }^{24}$ A consequence of this

TABLE IV. Corresponding structures of Figs. 3 and 4. The structure 1 (lowest formation energy for $25 \%$ and $75 \%$ ) is shown in Table III.

\begin{tabular}{|c|c|c|c|c|c|}
\hline Structure & Unit cell & $\begin{array}{l}25 \% \\
\text { No. }\end{array}$ & $\Delta H(\mathrm{meV})$ & $\begin{array}{r}75 \% \\
\text { No. }\end{array}$ & $\Delta H(\mathrm{meV})$ \\
\hline & $2 \times 2 \times 2$ & 2 & 76 & 2 & 56 \\
\hline & $2 \times 2 \times 1$ & 3 & 77 & 3 & 63 \\
\hline $40^{\circ}$ & $2 \times 1 \times 2$ & 4 & 83 & 5 & 88 \\
\hline 1800 & $2 \times 1 \times 1$ & 5 & 86 & 4 & 83 \\
\hline 40 & $2 \times 1 \times 2$ & 6 & 98 & 7 & 102 \\
\hline 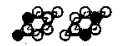 & $2 \times 1 \times 2$ & 7 & 103 & 6 & 97 \\
\hline 40 & $1 \times 1 \times 2$ & 8 & 109 & 8 & 138 \\
\hline
\end{tabular}

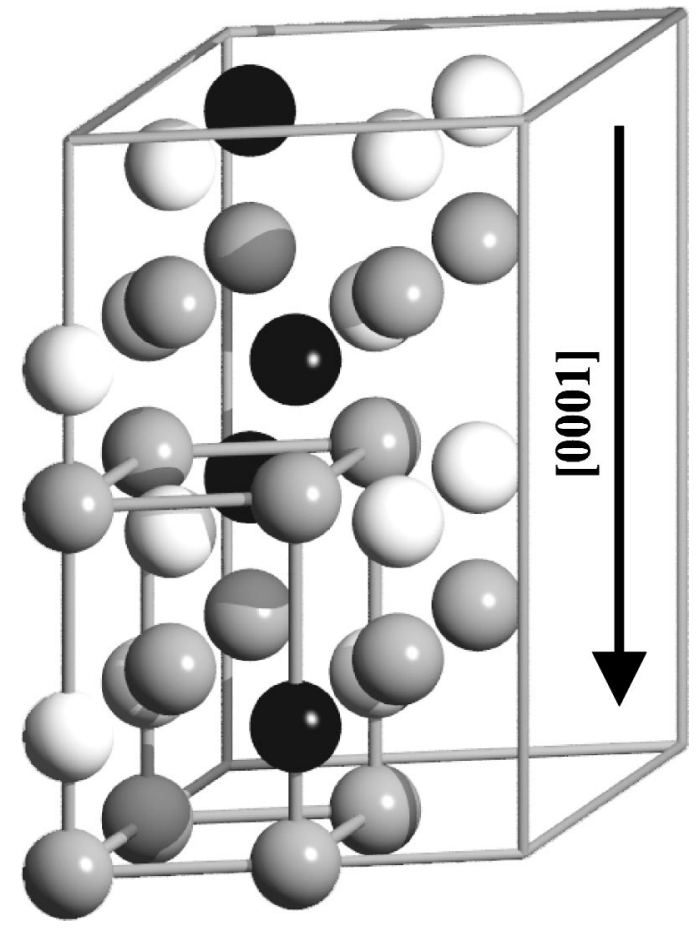

FIG. 1. Ordered structure with $25 \%$ (75\%) In. The wurtzite (2 $\times 2 \times 2$ ) unit cell contains $12 \mathrm{Ga}(\mathrm{In})$ atoms (white balls), $4 \mathrm{In}(\mathrm{Ga})$ atoms (black balls) and $16 \mathrm{~N}$ atoms (gray balls).

behavior is that [0001] superlattices (structure 8 in Figs. 3 and 4), i.e., stacked layers of pure $\mathrm{GaN}$ and $\mathrm{InN}$ along [0001], are energetically unfavorable. A spontaneous formation of [0001] superlattice structures is therefore thermodynamically forbidden. However, recent studies ${ }^{26}$ indicate that kinetic effects might stabilize this special ordered structure. A more detailed analysis of the formation energies and driving forces will be given elsewhere.

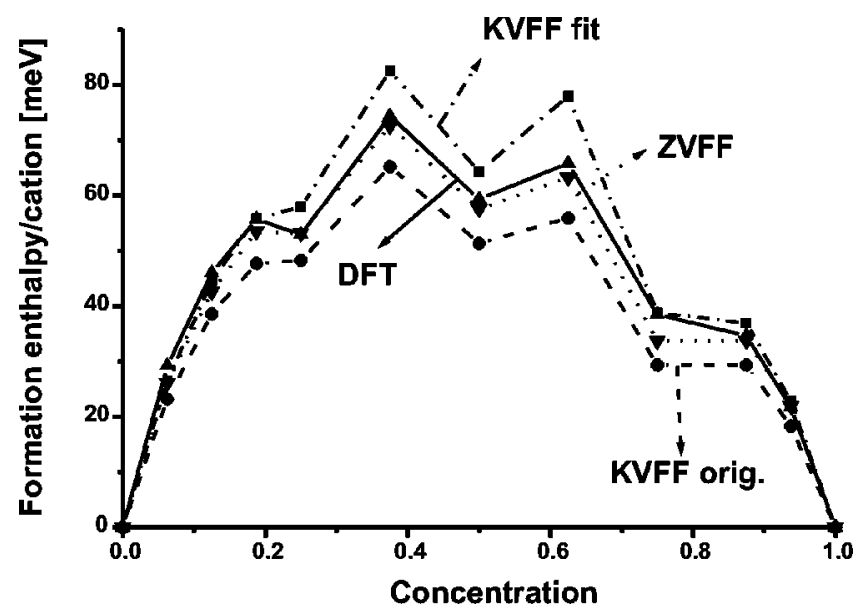

FIG. 2. The formation enthalpy for ordered structures (up to 32 atoms) in [meV/cation]. Only the structure with the lowest formation energy for a given concentration is plotted. (a) DFT calculations, (b) KVFF with parameter determination from $C_{11}^{c}$ and $C_{12}^{c}$, (c) KVFF with $C_{11}^{c}, C_{12}^{c}$, and $C_{44}^{c}$, (d) ZVFF. See also Table III, Table VI, and text. 


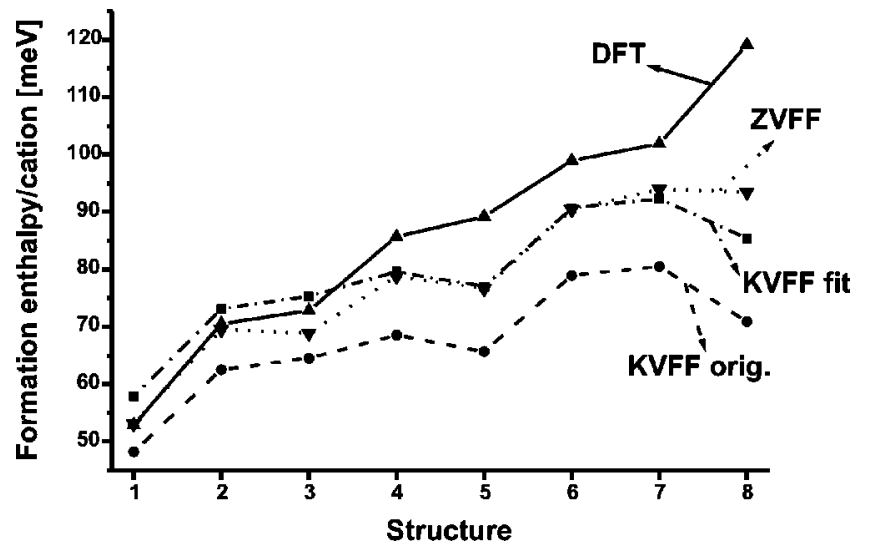

FIG. 3. The formation enthalpy of ordered structures (up to 32 atoms) in [meV/cation] for the concentration of $25 \%$. The structures differ in the local arrangement of the cations. (a) DFT calculations, (b) KVFF with parameter determination from $C_{11}^{c}$ and $C_{12}^{c}$, (c) KVFF with $C_{11}^{c}, C_{12}^{c}$, and $C_{44}^{c}$, (d) ZVFF. See also Table IV, Table VI, and text.

\section{VALENCE FORCE FIELD MODELS}

As has been pointed out in the Introduction, VFF models have been widely used to describe alloy properties such as bond lengths and formation energies. A detailed test about the accuracy and reliability of these methods for the highly ionic group III nitrides is lacking. We will therefore contrast our ab initio results (Sec. II) with commonly used VFF models. The starting point for almost all VFF models used to describe $\mathrm{In}_{x} \mathrm{Ga}_{1-x} \mathrm{~N}$ (Refs. 11-13) is the form given by Keating (KVFF),

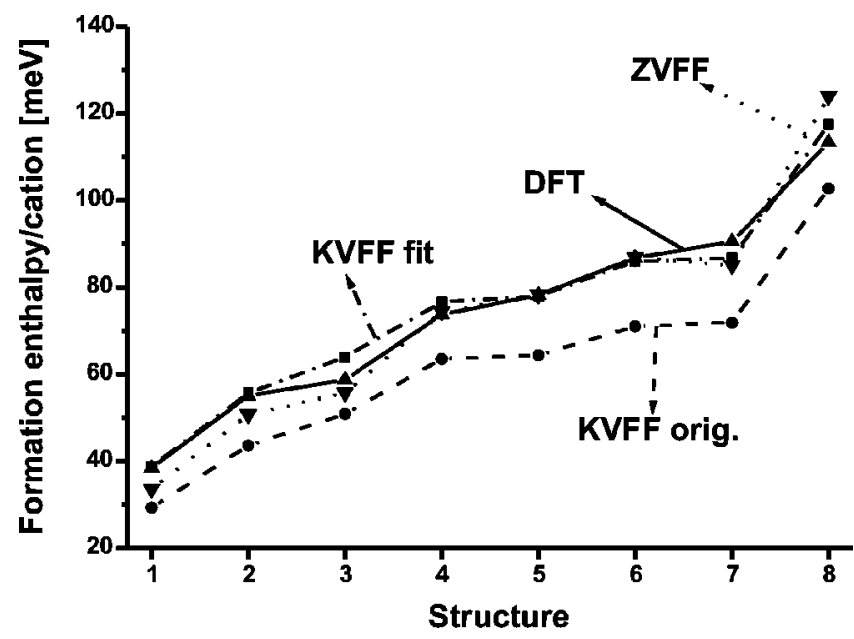

FIG. 4. The formation enthalpy of ordered structures (up to 32 atoms) in [meV/cation] for the concentration of $75 \%$. The structures differ in the local arrangement of the cations. (a) DFT calculations, (b) KVFF with parameter determination from $C_{11}^{c}$ and $C_{12}^{c}$, (c) KVFF with $C_{11}^{c}, C_{12}^{c}$, and $C_{44}^{c}$, (d) ZVFF. See also Table IV, Table VI, and text.

$$
\begin{aligned}
U^{\mathrm{VFF}}= & \sum_{s=1}^{N}\left\{\sum _ { i = 1 } ^ { 4 } \left[\frac{3}{4 d_{s i}^{2}} \alpha_{s i}^{2}\left(\mathbf{r}_{s i} \cdot \mathbf{r}_{s i}-d_{s i}^{2}\right)^{2}\right.\right. \\
& \left.\left.+\sum_{j=1, j \neq i}^{4} \frac{3}{8 d_{s i} d_{s j}} \beta_{i s j}\left(\mathbf{r}_{s i} \cdot \mathbf{r}_{s j}-d_{s i} d_{s j} \cos \left(\Theta_{i s j}\right)\right)^{2}\right\}\right]
\end{aligned}
$$

The strain energy $U^{\mathrm{VFF}}$ is calculated by a summation over the $N$ atoms of the unit cell. Only the next neighbors $i, j$ of atom $s$ are included in the interaction. Their actual distance vector is given by $\mathbf{r}$ with its equilibrium length $d$. The electronic part of the system is implicitly contained in the effective bond stretching parameter $\alpha_{s i}$, describing the two body interaction between the atoms $s$ and $i$, and the bond bending $\beta_{i s j}$ force constants, describing the three body interaction of atoms $i, s, j$ which in equilibrium form a common angle $\Theta_{i s j}$, around $s$.

The advantage of the formulation by Keating is that the three unknown parameters $d, \alpha$, and $\beta$ can be easily derived from the corresponding bulk systems: $d$ is the ideal bond length, $\alpha$ and $\beta$ can be readily calculated from the elastic constants [see Eq. (8) with $S=0$ ]. Based on these parameters we have calculated the equilibrium structure and the formation enthalpy for all ordered alloys. The procedure to find the equilibrium geometry is analogous to the first principles calculations, i.e., for each ordered structure both the external lattice parameters and the internal atomic positions are fully relaxed. The elastic bond bending parameter is chosen as the arithmetic average of the binary constituents

$$
\beta_{i s j}=\frac{\beta_{i s i}+\beta_{j s j}}{2}
$$

The results are shown in Fig. 2 for the energetically most stable structures and in Figs. 3 and 4 for different structures but the same composition. A comparison with the $a b$ initio calculated results shows that the Keating model gives a qualitative agreement. Quantitatively, however, significant differences are found: the formation energies for alloys are significantly underestimated. The average error in the formation energies is about $14.2 \mathrm{meV}$ per cation which is large compared with the formation enthalpies (see Table III).

In order to reduce the error we have checked several approaches. One problem with the Keating model is that it has only three variable parameters whereas the elastic properties of the bulk system are described by four parameters: the three elastic constants $\left(C_{11}^{C}, C_{12}^{C}, C_{44}^{C}\right)$ and the lattice constant. Therefore, as described above, only two elastic constants $\left(C_{11}^{C}, C_{12}^{C}\right)$ are commonly used to determine the Keating parameters. ${ }^{7,17}$ An important check for the validity of the Keating model is the calculation of the third elastic constant $C_{44}^{C}$. Within the Keating model $C_{44}^{C}$ can be easily expressed as

$$
C_{44}^{c}=\frac{\sqrt{3}}{r_{0}} \frac{\alpha \beta}{\alpha+\beta},
$$


TABLE V. Comparison of different valence force field (VFF) approaches for calculating the $C_{44}^{c 0}$ and $C_{44}^{c}$ constant (without and with internal atomic relaxation; see text) of the zincblende form. Ratios are given with respect to the DFT calculation. KVFF, Keating (Ref. 16); MVFF, Martin (Ref. 17); ZVFF, present.

\begin{tabular}{ccccc}
\hline \hline Compound & Ratio & KVFF & MVFF & ZVFF \\
\hline $\mathrm{GaN}$ & $C_{44}^{c 0}(\mathrm{VFF}) / C_{44}^{c 0}(\mathrm{DFT})$ & 1.22 & 1.21 & 1.15 \\
$\mathrm{GaN}$ & $C_{44}^{c}(\mathrm{VFF}) / C_{44}^{c}(\mathrm{DFT})$ & 0.69 & 0.74 & 0.75 \\
$\mathrm{InN}$ & $C_{44}^{c 0}(\mathrm{VFF}) / C_{44}^{c 0}(\mathrm{DFT})$ & 1.22 & 1.20 & 1.11 \\
$\mathrm{InN}$ & $C_{44}^{c}(\mathrm{VFF}) / C_{44}^{c}(\mathrm{DFT})$ & 0.60 & 0.73 & 0.75 \\
\hline \hline
\end{tabular}

As can be seen in Table $\mathrm{V}$, the deviation from the $a b$ initio value is rather large ( $69 \%$ for $\mathrm{GaN}, 60 \%$ for $\mathrm{InN})$. Trying to get a better agreement with the first principles calculations for the formation energies, we therefore use all three elastic constants to obtain the two Keating parameters $(\alpha, \beta)$ by a least squares fit. The parameters obtained following this procedure are listed in Table VI. Using these parameters we find a significantly reduced error of the formation energies for the ordered structures compared with our first principles calculations. The average error is $5.8 \mathrm{meV}$, i.e., only $41 \%$ of the initial error.

An inherent problem of the Keating model is the impossibility to distinguish between the zincblende and wurtzite structure. The reason is that this model takes only nearest neighbor interaction into account. The wurtzite and zincblende structures are, however, identical up to third nearest neighbors. As a consequence, the Keating model gives the same formation energy for the wurtzite and zincblende bulk phase. It also fails to describe nonideal $c / a$ ratios and the formation of two inequivalent bond lengths. This behavior is independent of how the $\alpha, \beta$ parameters are chosen. Mattila and Zunger therefore introduced two different bond lengths for the wurtzite lattice. ${ }^{12}$ Because we want to describe both phases within one and the same model we choose a different way to treat this problem (see Sec. IV).

A further issue is regarding the role of electrostatic interactions. A unique feature of group III nitrides compared to traditional semiconductors is their large ionicity, which has important consequences for the formation of, e.g., surfaces or defects. $^{27,28}$ While in binary bulk systems the electrostatic interaction can be partially described by the next nearest neighbor coupling within the Keating parameters this is not

TABLE VI. Model parameters for the different approaches. (b) Original Keating model (KVFF) with $S=0$ and parameters determined from $C_{11}^{C}$ and $C_{12}^{C}$. (c) Original Keating model with $S=0$ and parameters fitted to $C_{11}^{C}, C_{12}^{C}$, and $C_{44}^{C}$ (KVFF fit). (d) Present model (ZVFF). For further details see text.

\begin{tabular}{lccc}
\hline \hline & KVFF & KVFF fit & ZVFF \\
\hline$\alpha_{\mathrm{Ga}, \mathrm{N}}[\mathrm{N} / \mathrm{m}]$ & 84.2 & 81.4 & 97.4 \\
$\beta_{\mathrm{Ga}, \mathrm{N}, \mathrm{Ga}}[\mathrm{N} / \mathrm{m}]$ & 13.6 & 17.1 & 13.7 \\
$\alpha_{\mathrm{In}, \mathrm{N}}[\mathrm{N} / \mathrm{m}]$ & 68.9 & 66.3 & 78.6 \\
$\beta_{\mathrm{In}, \mathrm{N}, \mathrm{In}}[\mathrm{N} / \mathrm{m}]$ & 6.7 & 9.6 & 6.6 \\
\hline \hline
\end{tabular}

possible for ternary alloys where the specific arrangement of cations determines the electrostatic energy. We will therefore study in the following how the Keating model can be extended to account for electrostatic interactions.

\section{KEATING MODEL FOR HIGHLY IONIC COMPOUNDS}

The extension of the Keating model ${ }^{16}$ to include electrostatic interactions has been first developed by Martin. ${ }^{17}$ Martin included the Coulomb interaction by assuming a rigid point ion model (MVFF). Polarization effects have not been included: the effective charges $\mathrm{Ze}$ on the atoms are kept constant. The total energy of the system can then be written as the sum of the VFF strain energy [Eq. (2)] and the Coulomb energy $U^{\mathrm{C}}$

$$
U^{\mathrm{C}}=\frac{1}{2} \sum_{l \mu \nu} \frac{Z_{\mu} Z_{\nu} e^{2}}{\epsilon_{0}\left|\mathbf{r}_{s}^{l 0, \mu \nu}\right|}
$$

including the static electric constants. The distance between the $\mu$ th ion of the unit cell at the origin and the $\nu$ th ion in unit cell $l$ is represented by $\mathbf{r}_{s}^{l 0, \mu \nu}$. The Coulomb energy $U^{\mathrm{C}}$ can be easily evaluated following the Ewald scheme: the sum is split in a long- and short-range part, where the long-range part is evaluated in the reciprocal space and the short-range part in real space. ${ }^{29,30}$ For binary compounds with equal nearest neighbor bond length $r_{0}$ the Coulomb energy according to Eq. (5) can be simplified:

$$
U^{\mathrm{C}}=\alpha_{M} \frac{Z^{2} e^{2}}{\epsilon_{0} r_{0}} .
$$

The Madelung constant $\alpha_{M}$ is dependent on the crystal structure. Its numerical value is smaller for wurtzite $\left(\alpha_{M}^{\mathrm{WZ}}\right.$ $=-1.641)$ than for zincblende lattices $\left(\alpha_{M}^{\mathrm{WZ}}=-1.638\right)$. Therefore the stable crystal lattice of highly ionic group III nitrides is the wurtzite structure. It is important to note that by adding the Coulomb energy to the VFF model the parameter $d$ in Eq. (2) can no longer be set to the equilibrium bond length $r_{0}$. The Coulomb energy monotonically decreases with increasing lattice parameter resulting in an increased $d$ compared to the equilibrium bond length $r_{0}$. Using Eqs. (2) and (5) the dependence between $r_{0}$ and $d$ for zincblende systems can be readily evaluated:

$$
d^{2}=\frac{2(3 \alpha+\beta) r_{0}^{5}}{2(3 \alpha+\beta) r_{0}^{3}+\frac{Z^{2} e^{2}}{\epsilon} \alpha_{M}^{\mathrm{ZB}}}
$$

From Eq. (7) it is obvious that for weakly ionic systems $r_{0}$ $\approx d$. For strongly ionic systems, however, this difference can be significant. To be more specific, the corresponding values for $\mathrm{GaN}$ are $r_{0}=1.96 \AA$ and $d=1.99 \AA$, for $\operatorname{InN} r_{0}=2.17 \AA$ and $d=2.24 \AA$.

In his original approach Martin ${ }^{17}$ approximated the parameter $d$ by the equilibrium bond length $r_{0}$ and applied the method to weakly ionic compounds. Based on the above discussion, we expect this approximation to fail for more ionic compounds like group III nitrides. We have therefore ex- 
tended the original approach of Martin by taking the change in the parameter $d$ into account. Then, the relations between the Keating parameters $\alpha$ and $\beta$ and the elastic constants in our model are given by

$$
\begin{gathered}
C_{11}^{C}+2 C_{12}^{C}=\frac{\sqrt{3}}{4 r_{0}}(3 \alpha+\beta)+\left(F_{M}^{(1)}+F_{X}^{(1)}\right) S C_{0}, \\
F_{M}^{(1)}=\frac{\sqrt{3}}{8} \alpha_{M}^{\mathrm{ZB}} \approx-0.355, \\
F_{X}^{(1)}=\frac{\sqrt{3}}{16} 3 \alpha_{M}^{\mathrm{ZB}} \approx-0.532, \\
C_{11}^{C}-C_{12}^{C}=\frac{\sqrt{3}}{r_{0}} \beta+\left(F_{M}^{(2)}+F_{X}^{(2)}\right) S C_{0}, \\
F_{M}^{(2)} \approx 0.053, \\
F_{X}^{(2)}=\frac{\sqrt{3}}{2} \alpha_{M}^{\mathrm{ZB}} \frac{\beta}{3 \alpha+\beta} \approx 1.42 \frac{\beta}{3 \alpha+\beta}, \\
C_{0}=\frac{e^{2}}{r_{0}^{4}} .
\end{gathered}
$$

The above formulas contain the original Keating model by choosing the charge parameter $S=Z^{2} / \epsilon_{0}=0$, where $Z$ represents the ionic charge and $\epsilon$ the dielectric constant. The $F_{M}^{(1)}$ and $F_{M}^{(2)}$ are the corrections given by Martin with respect to the original Keating model. $F_{X}^{(1)}$ and $F_{X}^{(2)}$ represent the additional corrections if the change of the $d$ parameter is considered. From Eqs. (7) and (8) all parameters can be determined except for the charge parameter $S$. The standard way to determine the charge parameter $S$ is fitting to the $\omega_{\mathrm{LO}}-\omega_{\mathrm{TO}}$ phonon splitting which includes dynamical properties. ${ }^{7,17} \mathrm{We}$ are mainly interested in the formation energies which are static properties of the alloy systems. Therefore we use a different approach. If the energy difference per cation between the total energy of zincblende and ideal wurtzite structure (i.e., $c / a=\sqrt{8 / 3}$ and no internal relaxation) is given by $\Delta E_{\mathrm{tot}}^{\mathrm{id}}=E_{\mathrm{tot}}^{\mathrm{ZB}}-E_{\mathrm{tot}}^{\mathrm{WZ} \text {,id }}$ and using for both structures the same nearest-neighbor distance $r_{0}$ then the charge parameter $S$ can be readily evaluated using Eq. (6),

$$
S=\frac{r_{0} \Delta E_{\mathrm{tot}}^{\mathrm{id}}}{e^{2}\left(\alpha_{M}^{\mathrm{ZB}}-\alpha_{M}^{\mathrm{WZ}}\right)} .
$$

Using the calculated values for $\mathrm{GaN}\left(\Delta E_{\mathrm{tot}}^{\mathrm{id}}=10 \mathrm{meV}\right)$ and $\operatorname{InN}\left(\Delta E_{\mathrm{tot}}^{\mathrm{id}}=18 \mathrm{meV}\right)$ we obtain $S=0.41$ and $S=0.84$, respectively.
The parameters $\alpha$ and $\beta$ given in Table III have been calculated solely on the basis of the zincblende binary bulk systems: no information about the structural parameters of the wurtzite modification has been included. As a first test of our model — which we will call ZVFF-the bulk parameters for the wurtzite modification have been calculated (see Table II). They agree with the $a b$ initio calculated and experimental values for $\mathrm{GaN}$ as well as for $\mathrm{InN}$. In particular we note that the nonideality of the $c / a$ ratio and the existence of two inequivalent bond lengths are correctly reproduced.

As a second check we calculated the third elastic constant $\left(C_{44}\right)$ for the zincblende structure which has not been used to fit the parameters. Here we considered two cases: (i) no internal relaxation $C_{44}^{C 0}$ and (ii) full relaxation of internal coordinates $\left(C_{44}^{C}\right)$. Whereas case (ii) only gives slight improvements, the $C_{44}^{C 0}$ constant is described significantly better with the ZVFF compared to the original Keating model and to Martin's approach indicating that both internal and external strain effects are well described.

Finally we have applied the ZVFF model to determine the equilibrium structures and formation enthalpies of ordered $\mathrm{In}_{x} \mathrm{Ga}_{1-x} \mathrm{~N}$ structures in the same way as the KVFF and fitted KVFF. An averaged $S=0.63$ value is used to avoid discrepancies originating from polarization on the nitrogen, surrounded by Ga and In atoms.

The resulting formation enthalpies are shown in Figs. 2-4 and compared with our $a b$ initio, the KVFF and fitted KVFF calculations discussed already in the preceding section. Overall we find strongly improved formation enthalpies compared to the original KVFF and a further improvement compared to the "fitted" Keating model. The average errors for the three methods compared to the ab initio results are $14.2 \mathrm{meV}$ (KVFF), $5.8 \mathrm{meV}$ ("fitted" Keating), and 4.9 $\mathrm{meV}$ (ZVFF).

The main advantage of the presented VFF models is the small computational cost compared with the first principles calculations. A sufficient accuracy is possible by consistent determination of their parameters. Therefore the fitted KVFF and the ZVFF are suitable for strain relaxation in large cells with more than 1000 atoms and as tools for atomic prerelaxation in first principles calculations. The ZVFF model furthermore includes, in one and the same model, the correct energetic difference between the zincblende and wurtzite modification, gives the correct equilibrium wurtzite lattice parameters, and describes the $\operatorname{In}_{x} \mathrm{Ga}_{1-x} \mathrm{~N}$ ordered structures much better than the fitted KVFF model.

\section{CONCLUSIONS}

Based on a large set of ab initio calculated $\operatorname{In}_{x} \mathrm{Ga}_{1-x} \mathrm{~N}$ alloys we have studied the reliability and accuracy of various empirical VFF models. Using the original Keating model with standard parameter determination we find rather large differences in the formation energies. A significant improvement can be achieved by replacing the standard scheme to determine the Keating parameters by a least squares fit using all three zincblende elastic constants. Nevertheless, the correct description of nonideal wurtzite crystals can only be 
achieved by taking into account more than nearest neighbor interaction. For group-III nitrides, which are rather ionic, we find that the long-range interaction can be well approximatedby a simple point charge model. Despite its simplicity the ZVFF model correctly describes the differences between the wurtzite and zincblende phase, the formation energies, and atomic relaxation of $\operatorname{In}_{x} \mathrm{Ga}_{1-x} \mathrm{~N}$ alloys. Since the ZVFF model requires as input parameter only the elastic constants

*Author to whom correspondence should be addressed: Contact information HRL Laboratories, 3011 Malibu Canyon Road, Malibu, CA 90265. FAX: +01 310317 5679; Email: fgrosse@math.ucla.edu

${ }^{1}$ R. Singh, D. Doppalapudi, T. D. Moustakas, and L. T. Romano, Appl. Phys. Lett. 70, 1089 (1997).

${ }^{2}$ A. Wakahara, T. Tokuda, X.-Z. Dang, S. Noda, and A. Sakaki, Appl. Phys. Lett. 71, 906 (1997).

${ }^{3}$ N. A. El-Masry, E. L. Piner, S. X. Liu, and S. M. Bedair, Appl. Phys. Lett. 72, 40 (1998).

${ }^{4}$ D. Doppalapudi, S. N. Basu, and T. D. Moustakas, J. Appl. Phys. 85, 883 (1999).

${ }^{5}$ A. F. Wright and J. S. Nelson, Phys. Rev. B 51, 7866 (1995).

${ }^{6}$ A. F. Wright, J. Appl. Phys. 82, 2833 (1997).

${ }^{7}$ K. Kim, W. R. L. Lambrecht, and B. Segall, Phys. Rev. B 53, 16310 (1996).

${ }^{8}$ M. van Schilfgaarde, A. Sher, and A.-B. Chen, J. Cryst. Growth 178, 8 (1997).

${ }^{9}$ F. Bernardini, V. Fiorentini, and D. Vanderbilt, Phys. Rev. B 56, R10 024 (1997).

${ }^{10}$ C. Stampfl and C. G. Van de Walle, Phys. Rev. B 59, 5521 (1999).

${ }^{11}$ I. H. Ho and G. B. Stringfellow, Appl. Phys. Lett. 69, 2701 (1996).

${ }^{12}$ T. Mattila and A. Zunger, J. Appl. Phys. 85, 160 (1999).

${ }^{13}$ L. Bellaiche and A. Zunger, Phys. Rev. B 57, 4425 (1998). of the zincblende binary compounds and the energy difference between the cubic and the wurtzite phase it can be easily applied to other ionic alloy systems.

\section{ACKNOWLEDGMENT}

We would like to thank the Dutsche Forschungsgemeinschaft (Schwerpunktprojekt Gruppe-III-Nitride) for financial support.

${ }^{14}$ K. Kim, W. R. L. Lambrecht, and B. Segall, Phys. Rev. B 56, 7018 (1997).

${ }^{15}$ A. Silverman, A. Zunger, R. Kalish, and J. Adler, Phys. Rev. B 51, 10795 (1995).

${ }^{16}$ P. N. Keating, Phys. Rev. 145, 637 (1966).

${ }^{17}$ R. M. Martin, Phys. Rev. B 1, 4005 (1970).

${ }^{18}$ P. Hohenberg and W. Kohn, Phys. Rev. 136, B864 (1964).

${ }^{19}$ M. Bockstedte, A. Kley, J. Neugebauer, and M. Scheffler, Comput. Phys. Commun. 107, 187 (1997).

${ }^{20}$ A. F. Wright and J. S. Nelson, Phys. Rev. B 50, 2159 (1994).

${ }^{21}$ N. Troullier and J. L. Martins, Phys. Rev. B 43, 1993 (1991).

${ }^{22}$ M. Fuchs and M. Scheffler, Comput. Phys. Commun. 116, 1 (1999).

${ }^{23}$ H. J. Monkhorst and J. D. Pack, Phys. Rev. B 13, 5188 (1976).

${ }^{24} \mathrm{~F}$. Grosse and J. Neugebauer, in Proceedings of the 24th ICPS, Jerusalem 1998, edited by D. Gershoni (World Scientific, Singapore, 1999).

${ }^{25}$ F. Grosse and J. Neugebauer (unpublished).

${ }^{26}$ J. E. Northrup, L. T. Romano, and J. Neugebauer, Appl. Phys. Lett. 74, 2319 (1999).

${ }^{27}$ J. Neugebauer and C. G. Van de Walle, Festkoerperprobleme 35, 25 (1996).

${ }^{28}$ J. Neugebauer, T. Zywietz, M. Scheffler, J. E. Northrup, and C. G. Van de Walle, Phys. Rev. Lett. 80, 3097 (1998).

${ }^{29}$ P. P. Ewald, Ann. Phys. (Leipzig) 64, 253 (1921).

${ }^{30}$ E. R. Fuller and E. R. Naimon, Phys. Rev. B 6, 3609 (1972).

${ }^{31}$ S. Strite and H. Morkoc, J. Vac. Sci. Technol. B 10, 1237 (1992). 